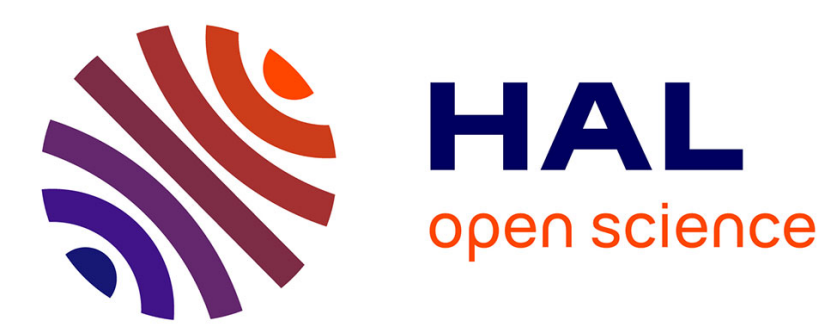

\title{
Experimental Study of the Flow in a Compact Heat Exchanger Channel With Embossed-Type Vortex Generators
}

\author{
Frédéric Dupont, Céline Gabillet, Patrick Bot
}

\section{- To cite this version:}

Frédéric Dupont, Céline Gabillet, Patrick Bot. Experimental Study of the Flow in a Compact Heat Exchanger Channel With Embossed-Type Vortex Generators. Journal of Fluids Engineering, 2003, 125 (4), pp.701-709. 10.1115/1.1595675 . hal-01582938

\section{HAL Id: hal-01582938 \\ https://hal.science/hal-01582938}

Submitted on 6 Sep 2017

HAL is a multi-disciplinary open access archive for the deposit and dissemination of scientific research documents, whether they are published or not. The documents may come from teaching and research institutions in France or abroad, or from public or private research centers.
L'archive ouverte pluridisciplinaire HAL, est destinée au dépôt et à la diffusion de documents scientifiques de niveau recherche, publiés ou non, émanant des établissements d'enseignement et de recherche français ou étrangers, des laboratoires publics ou privés. 


\title{
Experimental Study of the Flow in a Compact Heat
}

\section{Exchanger channel with Embossed-Type Vortex}

\section{Generators}

\author{
F. Dupont, C. Gabillet and P. Bot \\ Institut de Recherche de l'Ecole Navale F-29240 Brest-Naval, France \\ bot@ecole-navale.fr
}

10th February 2003

revised version

\begin{abstract}
The isothermal flow in a model channel of plate-fin heat exchanger with periodically arranged embossed-like vortex generators is investigated. Velocity measurements are performed by LDA in the transitional regime (Reynolds number from 1000 up to 5000). Strong longitudinal vortices are observed downstream of each generator. The vortex roll-up process is highlighted by the evolution of the velocity vector field in the cross section of the flow. The modifications of the vortex characteristics after successively encountered generators are investigated. This work shows most of the flow features which are known to produce heat transfer enhancement, and shows that these smooth shaped vortex generators are very promising for enhanced heat exchangers.
\end{abstract}




\section{Introduction}

To enhance heat transfer in industrial compact heat exchangers, an interesting passive method is to use longitudinal vortices in the flow. An industrial mean to generate such vortices is to place wings or winglets on the transfer surface, called vortex generators (for a review, see [1, 2]). It appears that a better knowledge of these vortex flows should enable a better design of optimized compact heat exchangers. A lot of work has been dedicated into analyzing the effect of vortex generators on the boundary layer of a single plate with a uniform flow far above the surface $[3,4,5]$. This enabled an insight into the mechanisms of longitudinal vortex induced transfer enhancement. It showed the interactions between the vortex and boundary layer [6] and the increase of turbulence intensity [7]. For example, Gentry and Jacobi [5] investigated the interactions of a vortex pair generated by delta wings with a boundary layer, and found a $50 \%$ to $60 \%$ enhancement of averaged transfer over a flat plate. However, for the issue of compact heat exchangers, it is necessary to extend this knowledge to the case of confined channel flows.

Investigation of confined channel flows with rectangular or delta wing and winglet vortex generators has recently motivated numerous numerical studies $[8,9,10,11]$ and a few experimental ones [12, 13]. A recent numerical study [14] has shown the efficiency of parallelepipedic generators. The strong confinement increases the number of involved parameters and the complexity of the flow. Thus, there is a real need in experimental studies to get a better insight into the transfer enhancement mechanism. In parametric studies $[2,9,10,15]$, recommendations for the design of vortex generators in compact heat exchangers are given. The geometrical configuration studied here (see section 2) is chosen according to these recommendations.

In a tube-fin exchanger, vortex generators can be obtained at reasonable manufacturing costs by punching the fins. The obtained triangular winglets perpendicular to the fins are known to give rise to a significant transfer enhancement $[13,15]$. In a plate-fin exchanger however, punching the fins is prohibited so as to avoid the mixing of both fluids. Due to the constraints in manufacturing costs, an acceptable method of obtaining vortex generators is to emboss the plates, which results in a smooth shaped winglet without sharp edges and of a limited height. The ability of such rounded embossed bodies to generate efficient vortices for transfer enhancement will be discussed.

The present work is an experimental investigation of the flow field in a channel of a vortex enhanced heat exchanger in a geometrical configuration close to industrial applications of plate-fin exchangers, where 
several vortex generators are periodically arranged. In order to be able to carry out flow measurements, the length scales are chosen to be around five times larger in the present experiment than in practical exchangers.

The aim of this work is to show the ability of embossed vortex generators to produce longitudinal vortices, and to precisely describe the resulting complex flow in a given geometry.

To our knowledge, the present are the first velocity measurements achieved in a channel with smooth obstacles simulating embossed vortex generators in a plate-fin exchanger. The downstream development of a vortex is investigated, as well as the evolution of the flow structure after the fluid successively encounters several vortex generators. The Reynolds number effects are also discussed. After a description of the experimental set-up, results of the velocity field and the vortex characteristics are presented. Finally, the expected effects on heat transfer are discussed, as well as how the present results may be of general interest for the issue of vortex enhanced heat exchangers.

\section{$2 \quad$ Experimental set-up}

The experiments have been conducted in a hydrodynamic loop made of a volumetric pump, two free surface buffer tanks, a test section and a by-pass system. The test section (label 1, on Fig. 1) consists of a $980 \mathrm{~mm}$ long, $860 \mathrm{~mm}$ high channel, with a width $H=27 \mathrm{~mm}$, delimited by lateral walls made of $25 \mathrm{~mm}$ transparent Perspex (2). In the upstream settling tank (3), diffusing plates (4), a honeycomb (5) and a converging section (6) are placed to homogenise the flow at the test section inlet. A mixing tank $(7)$ is placed downstream the test section which is fitted with mounting flanges (8). Flow rates are determined by a magnetic flow meter with a relative accuracy of $2 \%$. The experimental set-up enables to achieve a bulk velocity $U_{q}$ in the range from $0.037 \mathrm{~m} / \mathrm{s}$ to $0.37 \mathrm{~m} / \mathrm{s}$, corresponding to a Reynolds number $R e=\frac{U_{q} H}{\nu}$ in the range $[1000 ; 10000]$. The inlet velocity profile cannot be measured because there is no optical access at the entrance of the test section. Measurements in the channel without vortex generators were in reasonable agreement with the theory of developing flows in the entrance of a rectangular channel [16], and showed that the turbulence intensity was always lower than $5 \%$. At the beginning of the channel, the flow is considered as uniform and boundary layers develop downstream along the plates. In the rectangular test section, the flow in a plate-fin exchanger channel with embossed vortex generators is simulated. The vortex generators are wooden semi-cylinders which are attached to one of 
the lateral walls. Their length and height are respectively $2.7 \mathrm{H}$ and $0.37 \mathrm{H}$, and the ends are rounded with a curvature radius of $0.37 H$ in both directions ( $\frac{1}{4}$ of a sphere). Vortex generator pairs are arranged in four lines (streamwise horizontal direction : $\mathrm{x}$ ) and four columns (transverse vertical direction $\mathrm{y}$ ), as shown on Fig.2. In a pair, generators are symmetrically inclined with a $45^{\circ}$ angle of attack, separated by $1.3 H$. Lines are separated by $6.5 H$, and the distance between columns is $4 H$.

\section{Velocity measurements}

To investigate the flow in the channel, the longitudinal $u$ and vertical $v$ velocity components were measured by laser Doppler anemometry (LDA). Measurement of the spanwise velocity component $w$ is not possible in this experiment because of the orientation of the laser beams. A Dantec two component LDA system with three beams is used in the back scattering mode. A beam expander and a lens of $360 \mathrm{~mm}$ focal length provide a probe volume that is $0.5 \mathrm{~mm}$ long in the spanwise direction and $0.04 \mathrm{~mm}$ wide in the longitudinal and vertical directions. The probe volume is traversed in the flow by a mechanical displacement system of absolute accuracy of 5 microns. Thus, the uncertainty of the measurement position relative to the walls is $0.5 \mathrm{~mm}$. The water flow is seeded with Iriodine particles of typical size of one micron. The scattered light signal is processed by two Dantec Burst Spectrum Analyzers. The $u$ and $v$ velocity components have been recorded in a cross section of the flow over a grid of 20 nodes in the spanwise direction and 30 nodes in the vertical direction, with a $1.3 \mathrm{~mm}$ step. At each position, a maximum of 2500 data points are collected at a typical data rate of $50 \mathrm{~Hz}$, and the mean $(U, V)$ and $\mathrm{rms}\left(u^{\prime}, v^{\prime}\right)$ values are computed. $\mathrm{U}$ and $\mathrm{V}$ are estimated with a relative accuracy of $\pm 2 \%$ and $\pm 6 \%$ respectively with a confidence level of 95\%. Rms values are obtained with a relative accuracy of $\pm 3 \%$.

\section{Results}

The whole flow is expected to be symmetrical with respect to the streamwise center line of the test section. Moreover, the flow behind each vortex generator pair should be symmetric with respect to the center line of the pair. Velocity measurements are carried out in one cross-section downstream of each vortex generator on a single line just above the channel center line. To see the evolution of a vortex downstream of a generator, measurements have also been taken in three different cross sections behind the second column. The location of each measurement section $x$ is shown in Fig. 2 and summarized in 
Table 1, where the distance downstream of the generator $d$ is also given. Three flow rates have been studied, corresponding to $R e=1000,2000$ and 5000 (i.e.: $U_{q}=0.037,0.074$ and $0.188 \mathrm{~m} / \mathrm{s}$ ).

\begin{tabular}{|c|c|c|}
\hline$\frac{x}{H}$ & column number & $\frac{d}{H}$ \\
\hline \hline 7.1 & 1 & 2.4 \\
\hline 9.1 & & 0.4 \\
10.1 & 2 & 1.4 \\
11.1 & & 2.4 \\
\hline 15.1 & 3 & 2.4 \\
\hline 19.1 & 4 & 2.4 \\
\hline
\end{tabular}

Table 1: Summary of all measurement sections' reduced position $\frac{x}{H}$, column number and corresponding reduced distance behind the vortex generator trailing edge $\frac{d}{H}$.

To have a first insight into the flow structure, a visualization has been made by dye injection just upstream of the first vortex generator (Fig. 3). The dye line first follows the body suction side. Then a helical emission line appears after the separation point. This visualization shows the development of a vortex and its trajectory downstream of the vortex generator.

The evolution of the velocity field is investigated in detail behind the second column of vortex generators. Then, global vortex parameters are studied after a succession of generators.

\subsection{Velocity fields}

Firstly, the mean velocity components are investigated. Velocities are scaled by the bulk velocity $U_{q}$, and lengths are normalized by the channel width $H$. Using the measurement sections at $\frac{x}{H}=9.1,10.1,11.1$, it is possible to estimate $\frac{\partial U}{\partial x}$ and $\frac{\partial V}{\partial y}$ at the cross sections $\frac{x}{H}=9.6,10.1$ and 10.6, and then to determine the third component $W$ using the continuity equation (Eq. 1).

$$
\frac{\partial U}{\partial x}+\frac{\partial V}{\partial y}+\frac{\partial W}{\partial z}=0
$$

The derivatives have been computed by a finite difference method with a center scheme, and the $W$ component has been integrated by the trapezoid method. On the domain boundaries, forward and backward schemes have been used. A zero value has been applied to each velocity component on both walls (no slip no through boundary condition). Within this framework, if $U$ and $V$ measurements are 
considered as independent random variables, computation of the velocity gradient $\frac{\partial W}{\partial z}$ is assumed to be accurate to within $\frac{U_{q}}{H}$. Therefore, $W$ is estimated with an accuracy of $0.05 U_{q}$ near the walls. In the central part of the channel, uncertainty becomes higher, up to the order of $U_{q}$, because of integration along the $\mathrm{z}$ direction. In fact, computation of the finite differences should eliminate the systematic errors. Another source of error that cannot be estimated here comes from the estimation of space derivatives by discrete finite differences. Despite this observation of rather poor accuracy, computation of the velocity component $W$ has been used to get a qualitative idea of the velocity vector field and the vortex structure at three positions downstream of the second vortex generator.

Velocity fields in the three cross sections behind the second generator are shown on Fig. 4 for $V$ and Fig. 5 for $U$, for the three Reynolds numbers. Figure 6 shows the corresponding mean velocity vector field in the cross section.

The existence of an intense longitudinal vortex developing downstream of the generator is shown in the velocity fields by the positive and negative peaks facing each other in the $V$ distribution (Fig. 4), and by the longitudinal velocity deficit in the $U$ distribution (Fig. 5). The vortex structure is also clearly highlighted by the vector fields in the different cross sections (Fig. 6). With a rather circular appearance for the lower Reynolds number, the vortex is elongated in the y direction for $R e=5000$. Moreover, it is located closer to the wall where the generators are attached $\left(\frac{z}{H} \simeq-0.3\right)$, particularly for the higher Reynolds number values. It is also noticeable that the extrema of transverse velocity are located very close to the walls (Fig. 4). As a result, the velocity gradients on the walls are sharp, which implies a strong momentum transfer in transverse and spanwise directions.

For $R e=1000$ at the first two locations, the top half of another vortex appears in the bottom-righthand corner $\left(\frac{z}{H} \simeq-0.1 ; \frac{y}{H} \simeq-0.85\right)$. This small secondary vortex, rotating in the opposite direction, is induced by the main vortex in the vicinity of the wall. It is not actually visible for higher Reynolds numbers. This could be because it is out of the measurement area.

An important asymmetry in the $\mathrm{z}$ direction in the $V$ velocity distribution is observed for the three cross sections and for each Reynolds number. The negative peak of $V$ is much stronger than the positive one, with $V_{\min } \simeq-0.5 U_{q}$ to $-0.8 U_{q}$ near the wall where the generators are attached, and $V_{\text {max }} \simeq 0.2$ to $0.3 U_{q}$ near the opposite wall. This asymmetry is stronger for a higher Reynolds number, and may be related to two causes. It results from the interaction between the vortex and the neighboring wall, as predicted by the image theory [5, 17]. But it is also an effect of a downward deviation of the flow 
due to the body orientation. As shown by Figure 3 for $R e=2000$, vortices are oriented with an angle approximately equal to half the body angle of attack, showing the flow deviation and vortices drifting along the wall.

In addition, the signature of the longitudinal vortex roll up is shown by the longitudinal, $u$, velocity field (Fig. 5). At $\frac{x}{H}=9.1$, the longitudinal velocity presents a large area of low velocity near the wall where the bodies are attached. This region corresponds to the wake of the body. The vortex formation progressively appears by the generation of a velocity deficit in the vortex core, which reaches $0.45 U_{q}$. This "wake-like" effect results from the slowing down of the fluid by the body, and from the vortex development from $\frac{x}{H}=9.6$ to $\frac{x}{H}=10.1$. It is noticeable that the higher the Reynolds number, the earlier the vortex development. In fact, the local velocity deficit in the vortex core shows up at $\frac{x}{H}=10.1$ for $R e=2000$ and 5000, but hardly appears at $\frac{x}{H}=11.1$ for $R e=1000$.

A local acceleration (up to $1.1 U_{q}$ ) is shown outside the vortex, which verifies the mass conservation. Furthermore, a thinning of the boundary layer shows up where the vortex induced velocities are directed towards the wall, on the middle right-hand side and bottom left-hand corner, particularly for $\frac{x}{H} \geq 10.1$. These so-called downwash flow regions are responsible for a local increase in the mean momentum transfer with the wall. However, in the regions where the vortex induced velocities pull the fluid out of the wall (upwash flow regions : on the top left and bottom right-hand corners), the boundary layer is obviously thicker, leading to a decrease of local momentum transfer.

\subsection{Velocity fluctuations}

The iso-values of the rms longitudinal and vertical velocity $u^{\prime}$ and $v^{\prime}$ measured at $\frac{x}{H}=11.1$ are shown on Fig. 7. To determine whether or not these fluctuations are associated with a coherent motion in the body wake, a spectral analysis has been performed on the time series of the velocity measurements, using fast Fourier transform, after re-sampling of the LDA signals. As shown for example on Fig. 8, no significant periodicity has been detected in any velocity time series. Then, the rms velocity may be associated with turbulence, in the wide sense of random fluctuations.

An interesting point is the high level of the vertical fluctuating motion in the core of the vortex, $\frac{v^{\prime}}{U_{q}} \simeq 0.25$ (Fig. 7.a). Moreover, as a consequence of the blowing effect in the upwash region and a redistribution between the spanwise and vertical fluctuating components, a remarkable increase in $v^{\prime}$

can be seen (up to $\frac{v^{\prime}}{U_{q}} \simeq 0.25$ ). As it is expected in the region where the boundary layer is thinner, 
the longitudinal fluctuating velocity component is high $\left(\frac{u^{\prime}}{U_{q}} \simeq 0.25\right.$, Fig. $\left.7 . \mathrm{b}\right)$. Thus, an increase in the production of turbulent kinetic energy, due to the shear stresses under velocity gradients in these near wall regions, is expected to have a strong influence on the longitudinal component. Focusing our interest on the global influence of the Reynolds number and successive generators on turbulence, we consider the space averaged turbulent kinetic energy $k$. With the assumption that $w^{\prime} \simeq v^{\prime}, k$ may be estimated by $k=<\frac{1}{2}\left(u^{\prime 2}+2 v^{2}\right)>$, where angular brackets denote a space averaging over the whole measurement cross section. For $R e=1000$, the turbulent kinetic energy increases from $\frac{k}{\frac{1}{2} U_{q}^{2}}=0.03$ behind the first vortex generator $\left(\frac{x}{H}=7.1\right)$ to $\frac{k}{\frac{1}{2} U_{q}^{2}}=0.07$ behind the third and fourth vortex generators $\left(\frac{x}{H} \geq 15.1\right)$ (Fig. 9). For $R e \geq 2000$, the turbulent kinetic energy is neither sensitive to the Reynolds number nor to the successive generators encountered and reaches $\frac{k}{\frac{1}{2} U_{q}^{2}}=0.1$. These results demonstrate that the flow turbulence is developed after the first vortex generator for $R e \geq 2000$.

\subsection{Vortex parameters}

In this section, the global flow structure is analyzed from the recorded mean velocity fields in terms of the main vortex characteristics. The vortex core size and intensity are deduced from the $V$ velocity component in the following way. The mean vortex core radius $a$ is defined as half the distance between the positions of the maximum $V_{\max }$ and minimum $V_{\min }$ in the $V$ velocity distribution (see Fig. 4). The relative accuracy of $a$ is estimated to $\pm 15 \%$. The vortex maximum tangential velocity is estimated as $V_{t \max }=\frac{1}{2}\left(V_{\max }-V_{\min }\right)$, and the intensity of the vortex is defined as the circulation $\Gamma=2 \pi a V_{t \max }$, leading to an accuracy of $\pm 6 \%$ and $\pm 20 \%$ respectively for these two quantities. This method enables the determination of the vortex intensity even when the third velocity component $W$ is not measured. This intensity is related to the large scale mixing effect of the vortex.

The evolution downstream of one vortex generator and the modification of the vortex features with the successive bodies encountered ("column effect") are analyzed for different values of the Reynolds number. The maximum tangential velocity $V_{t \max }$ decreases downstream because of vortex dissipation (Fig. 10). As the transverse velocity extrema are located close to the walls, the dissipation is mainly due to friction on the wall, which results in a high momentum transfer to the wall. This is particularly true for the highest Reynolds number. However, at a distance $\frac{d}{H}=2.4$ behind the vortex generator, the maximal tengential velocity $\frac{V_{t \max }}{U_{q}} \simeq 0.45$ is nearly independent of $R e$. The vortex core size remains quite constant downstream of a generator, but the higher the Reynolds number, the larger the vortex (Fig. 11). This 
is a consequence of a stronger flow separation on the vortex generator for an increasing Re. In addition, the thinning of the boundary layer with Re allows a larger vortex to develop. The same conclusion holds for the circulation : it is clearly augmented by the increase of $R e$ and a decrease is observed downstream of each generator, down to $\frac{\Gamma}{U_{q} H}=0.72$ for $R e=1000$ and $\frac{\Gamma}{U_{q} H}=1.15$ for $R e=5000$, at $\frac{d}{H}=2.4$ behind the vortex generator (Fig. 12). Considering the successive generators encountered by the flow, there is no evidence of any variation of $V_{t \max }$. However, it is interesting to note that the size of each vortex increases from the first to the third column (Fig. 13). This fact indicates that the vortices of successive generators interact with each other, and that a vortex may benefit from vorticity generated upstream. Nevertheless, a decrease of the vortex size shows up beyond the third column. This observation may be attributed to the development of the boundary layer on the flat plate that limits the vortex size. A similar behavior to the vortex size is observed for the circulation (Fig. 14).

\section{Discussion}

Like any vortex generator, embossed bodies are dedicated to enhance heat transfer. Here, we discuss the possible effects on heat transfer of the vortices observed in the present isothermal flow. The first point is the vortex induced large scale mixing, and its role in heat advection. Indeed, the advective heat flux per unit surface $q$ depends on the temperature difference across the vortex $\Delta T$, and the maximum tangential velocity directly linked to the vortex intensity and size : $q=\rho C_{p} \frac{\Gamma}{2 \pi a} \Delta T$. According to Gentry and Jacobi [5], the location of the vortex core relative to the thermal boundary layer is the key factor for the advective heat transfer across the vortex. The vortex should not remain outside the boundary layer, in order to come across an area of strong temperature difference, leading to a high advected flux. In the present confined geometry, and considering the large size of observed vortices (from 0.5 up to $0.8 H$ ), there is no doubt that it would spread over an area of high temperature difference, in the corresponding heated flow. Hence, the advective flux due to the vortex is likely to be strong.

The second point is the boundary layer thinning observed in the downwash region. Experimental studies have shown that the thinning of the boundary layer is always associated with a local increase of heat transfer on the wall, both for turbulent [6] and laminar [7] boundary layers developing over a flat plate. It may be argued that this statement still holds in a confined channel.

The effect of the fluctuating motion on heat transfer is less straightforward. Yanagihara and Torii [7] 
suggested that the transfer enhancement observed in the upwash flow region may be linked to the increase

of turbulence intensity in this region, and that this effect extends far downstream of the generator.

Finally, the isothermal vortex flow investigated here reveals characteristic features which are known to enhance heat transfer. The efficiency of these embossed vortex generators to enhance transfer is supported by some preliminary results of a mass transfer study made by Schulz and Fiebig, with the ammonia absorption method, in a channel with similar embossed generators [18].

A systematic experimental study for different geometries was not possible at this stage, since geometric parameters are too numerous. Such a parametric study should be achieved either by numerical tools or through global heat transfer measurements. Moreover, searching for an optimized configuration should include pressure loss measurements and other economic considerations which may be different for each application. Nevertheless, the present experimental investigation should provide data for a better understanding of transfer enhancement mechanisms, and for the validation of numerical tools.

\section{Conclusion}

The flow in a confined channel with periodically arranged smooth-shaped vortex generators is investigated. The experiment is designed to simulate the flow in a channel of industrial plate-fin heat exchanger with embossed vortex generators. The mean and fluctuating velocity fields are characterized by LDA for different values of the Reynolds number. Measurements are performed in several cross sections behind the successive generators. The flow field shows the existence of strong longitudinal vortices behind each vortex generator, which are known to give rise to significant heat transfer enhancement [5, 6, 7]. Indeed, the large scale mixing is highlighted by the large size and intensity of vortices, and a noticeable thinning of the boundary layer is observed in the downwash flow regions. Moreover, local turbulent mixing is strong, particularly in the upwash and downwash flow regions and in the core of the vortices. Vortices are characterized by a high tangential velocity $\left(0.5\right.$ to $\left.0.8 U_{q}\right)$ and a wake-like deficit of streamwise velocity in the core. The non-dimensional vortex intensity increases with the Reynolds number due to a stronger flow separation. As vortices are located closer to the embossed wall, heat transfer is expected to be enhanced preferentially on this wall. An interesting result in the periodical arrangement of the generators is the contribution of the vorticity induced by the upstream generators to the increased vortex sizes and intensities from generators located downstream. However, saturation takes place after the third 
generator column. Moreover, the turbulence development is accelerated by vortex generators. In fact, for a high enough Reynolds number $(R e \geq 2000)$, the flow reaches its maximum turbulence level after the first vortex generator column. Overall, embossed vortex generators are shown to be a promising alternative to punched winglets for a plate-fin type heat exchanger.

\section{Acknowledgements}

This work was supported by the E.C. Joule Program contract JOE3-CT97-0056. The authors are grateful to MM. Mitra, Leiner, Fruman and Billard for valuable discussions, and to the referees for their interesting remarks.

\section{Nomenclature}

$a=$ mean vortex core radius, $\mathrm{m}$

$C_{p}=$ heat capacity of the fluid, $\mathrm{J} / \mathrm{kg} . \mathrm{K}$

$d=$ distance downstream of the body trailing edge, $\mathrm{m}$

$H=$ channel width, $\mathrm{m}$

$k=$ turbulent kinetic energy, $\mathrm{m}^{2} / \mathrm{s}^{2}$

$q=$ advective heat flux, $\mathrm{W} / \mathrm{m}^{2}$

$R e=$ Reynolds number $\left(=\frac{U_{q} H}{\nu}\right)$, dimensionless

$U=$ mean longitudinal velocity component, $\mathrm{m} / \mathrm{s}$

$U_{q}=$ bulk velocity, $\mathrm{m} / \mathrm{s}$

$u=$ longitudinal velocity component, $\mathrm{m} / \mathrm{s}$

$u^{\prime}=\mathrm{rms}$ longitudinal velocity component, $\mathrm{m} / \mathrm{s}$

$V=$ mean vertical velocity component, $\mathrm{m} / \mathrm{s}$

$V_{\min }=$ minimum of mean vertical velocity in a cross section, $\mathrm{m} / \mathrm{s}$

$V_{\max }=$ maximum of mean vertical velocity in a cross section, $\mathrm{m} / \mathrm{s}$

$V_{t \max }=$ maximum of tangential velocity in a cross section, $\mathrm{m} / \mathrm{s}$

$v=$ vertical velocity component, $\mathrm{m} / \mathrm{s}$

$v^{\prime}=\mathrm{rms}$ vertical velocity component, $\mathrm{m} / \mathrm{s}$

$W=$ mean spanwise velocity component, $\mathrm{m} / \mathrm{s}$ 
$w=$ spanwise velocity component, $\mathrm{m} / \mathrm{s}$

$w^{\prime}=$ rms spanwise velocity component, $\mathrm{m} / \mathrm{s}$

$x=$ streamwise coordinate, $\mathrm{m}$

$y=$ vertical coordinate, $\mathrm{m}$

$z=$ spanwise (normal to plates) coordinate, $\mathrm{m}$

\section{Greek Symbols}

$\Delta T=$ température difference across the vortex, $\mathrm{K}$

$\Gamma=$ vortex circulation, $\mathrm{m}^{2} / \mathrm{s}$

$\nu=$ kinematic viscosity of the fluid, $\mathrm{m}^{2} / \mathrm{s}$

$\rho=$ density of the fluid, $\mathrm{kg} / \mathrm{m}^{3}$

\section{References}

[1] A. M. Jacobi, R. K. Shah. Heat Transfer Surface Enhancement through the Use of Longitudinal Vortices : A Review of Recent Progress. Experimental Thermal Fluid Science, 11:295-709, 1995.

[2] M. Fiebig. Vortex Generators for Compact Heat Exchangers. Journal of Enhanced Heat Transfer, 2(1-2):43$61,1995$.

[3] I. M. M. A. Shabaka, R. D. Metha and P. Bradshaw. Longitudinal Vortices Embedded in Turbulent Boundary Layers. Part 1 Single Vortex. J. Fluid Mech, 155:37-57, 1985.

[4] R. D. Mehta and P. Bradshaw. Longitudinal Vortices Embedded in Turbulent Boundary Layers. Part 2 Vortex Pair with Common Flow Upwards. J. Fluid Mech, 188:529-546, 1988.

[5] M. C. Gentry and A. M. Jacobi. Heat Transfer Enhancement by Delta-Wing Vortex Generators on a Flat Plate : Vortex Interactions with the Boundary Layer. Experimental Thermal Fluid Science, 14:231-642, 1996.

[6] P. A. Eibeck, J. K. Eaton. Heat Transfer Effects of a Longitudinal Vortex Embedded in a Turbulent Boundary Layer. ASME, 109, February 1987.

[7] J. I. Yanagihara and K. Torii. Enhancement of Laminar Boundary Layer Heat Transfer by a Vortex Generator. JSME Int, 35(3):400-405, 1992. 
[8] M. Fiebig, N. Mitra and Y. Dong. Simultaneous Heat Transfer Enhancement and Flow Loss Reduction of Fin-Tubes. 9th Int Heat Transfer Conf, 9:51-55, August 1990.

[9] G. Biswas and H. Chattopadhyay. Heat Transfer in a Channel with Built-in Wing Type Vortex Generators. J. Heat Mass Transfer, 35(4):803-814, 1992.

[10] G. Biswas, K. Torii, D. Fujii and K. Nishino. Numerical and Experimental Determination of Flow Structure and Heat Transfer Effects of Longitudinal Vortices in a Channel Flow. J. Heat Mass Transfer, 39(16):3441$3451,1996$.

[11] M. Fiebig and N. K. Mitra. Development in Heat Transfer : Computer Simulation in Compact Heat Exchangers, volume 1, chapter 8, pages 220-254. Computational Mechanics Publications, B. Sunden M. Faghri edition, 1998.

[12] S. Lau. Experimental Study of the Turbulent Flow in a Channel with Periodically Arranged Longitudinal Vortex Generators. Experimental Thermal Fluid Science, 14:255-261, 1995.

[13] M. Fiebig, A. Valencia and N. K. Mitra. Wing-Type Vortex Generators for Fin-and-Tube Heat Exchangers. Experimental Thermal Fluid Science, 7:587-295, 1993.

[14] A. Sohankar, L. Davidson. Effect of Inclined Vortex Generators on Heat Transfer Enhancement in a ThreeDimensional Channel. Numerical Heat Transfer. Part A, 39(5):433-448, April 2001.

[15] A. Valencia, M. Fiebig and N. K. Mitra. Heat Transfer Enhancement by Longitudinal Vortices in a Fin-Tube Heat Exchanger Element with Flat Tubes. Journal of Heat Transfer, 118:209-211, February 1996.

[16] H. Schlichting. Boundary-Layer Theory. Classic TextBook Reissue. McGraw-Hill, 7th edition, 1979.

[17] M. Mokry, W. J. Rainbird. Calculation of Vortex Sheet Roll-up in a Rectangular Wind Tunnel. Journal of Aircraft, 12:750-752, 1975.

[18] K. Schulz, M. Fiebig. Accurate Inexpensive Local Heat/Mass Transfer Determination by Digital Image Processing Applied to the Ammonia Absorption Method. Private Communication, 2000. 


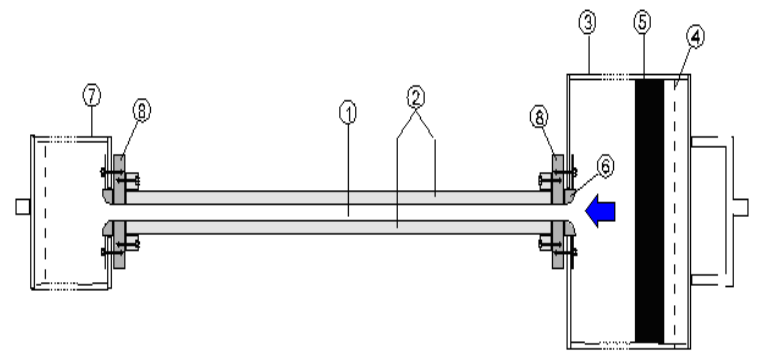

Figure 1: Top view of the experimental facility. 


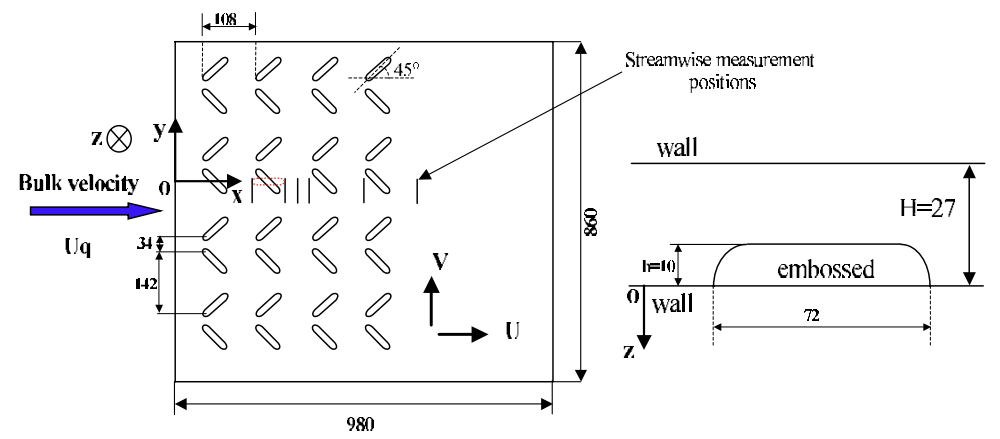

Figure 2: Geometry of the channel with mounted embossed-like vortex generators. All dimensions are in millimetres. Pairs of generators are arranged in four lines (streamwise horizontal direction : $x$ ) and four columns (transverse vertical direction y). 


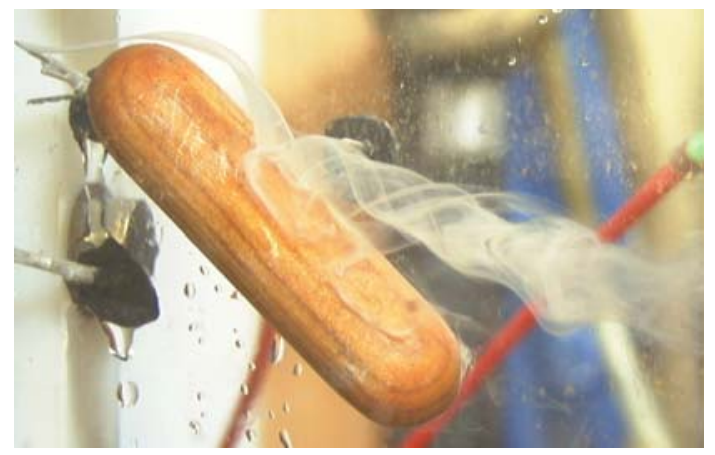

Figure 3: Vortex visualization by dye injection, for $R e=1000$, behind a generator of the first column. 

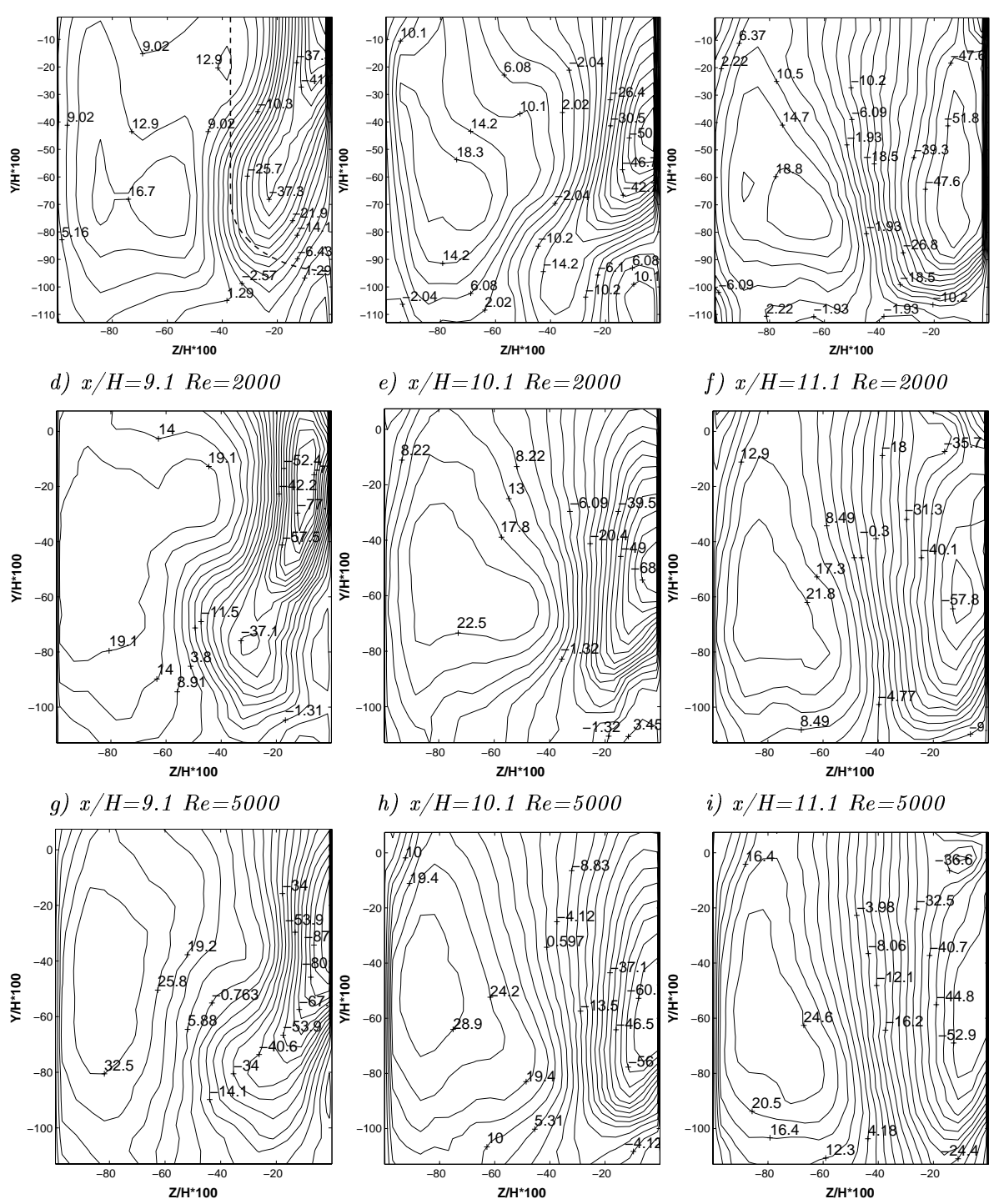

Figure 4: Iso-values (\%) of mean vertical velocity component $\frac{V}{U_{q}} \times 100$, for $R e=1000(a, b, c), R e=2000$ $(d, e, f)$ and $R e=5000(g, h, i)$ behind the $2^{\text {nd }}$ column at three streamwise cross sections $(O z, O y)$ 

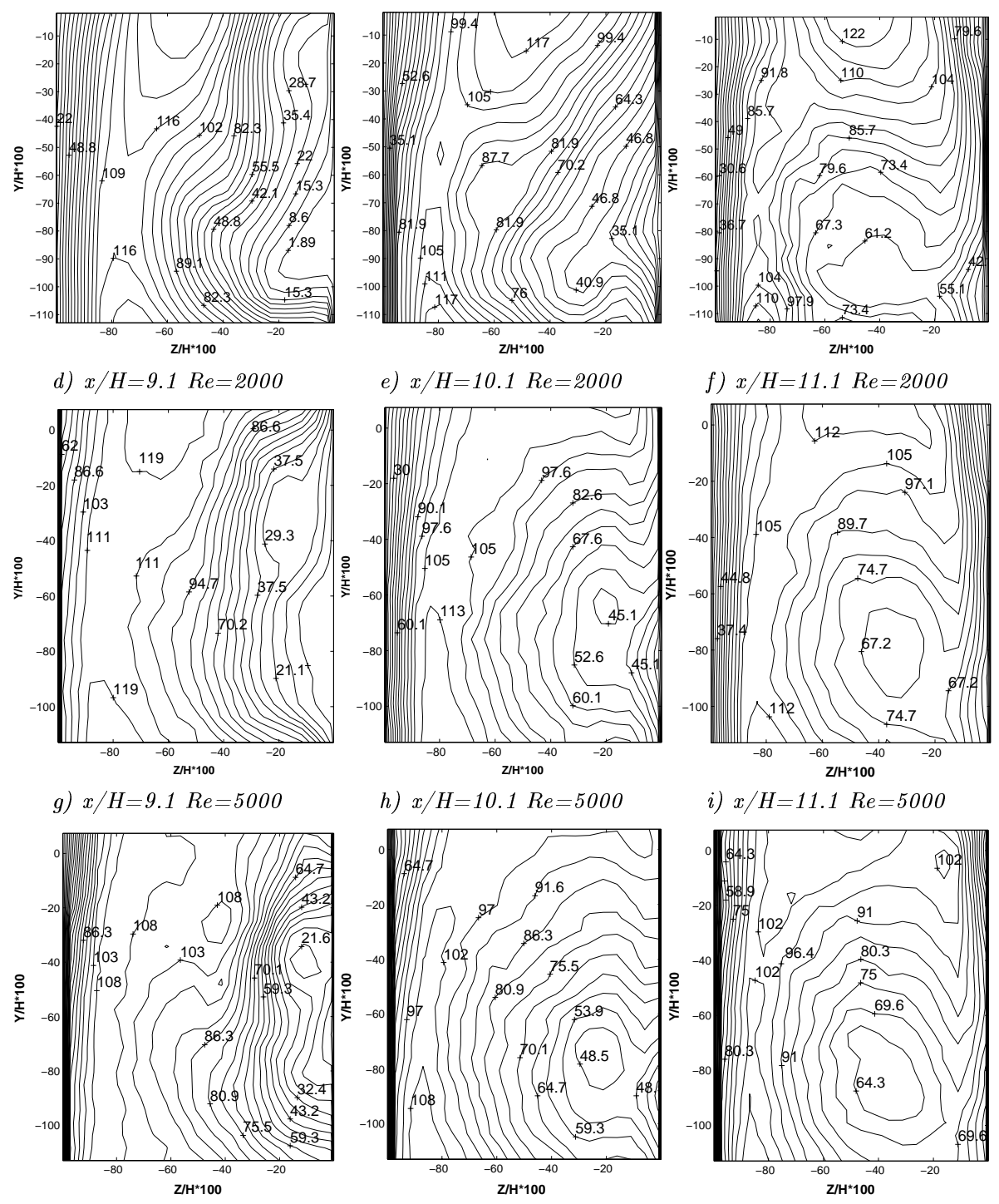

Figure 5: Iso-values (\%) of mean longitudinal velocity component $\frac{U}{U_{q}} \times 100$, for $\operatorname{Re}=1000$ (a, b, c), $R e=2000(d, e, f)$ and $R e=5000(g, h, i)$ behind the $2^{\text {nd }}$ column at three streamwise cross sections (Oz,Oy) 

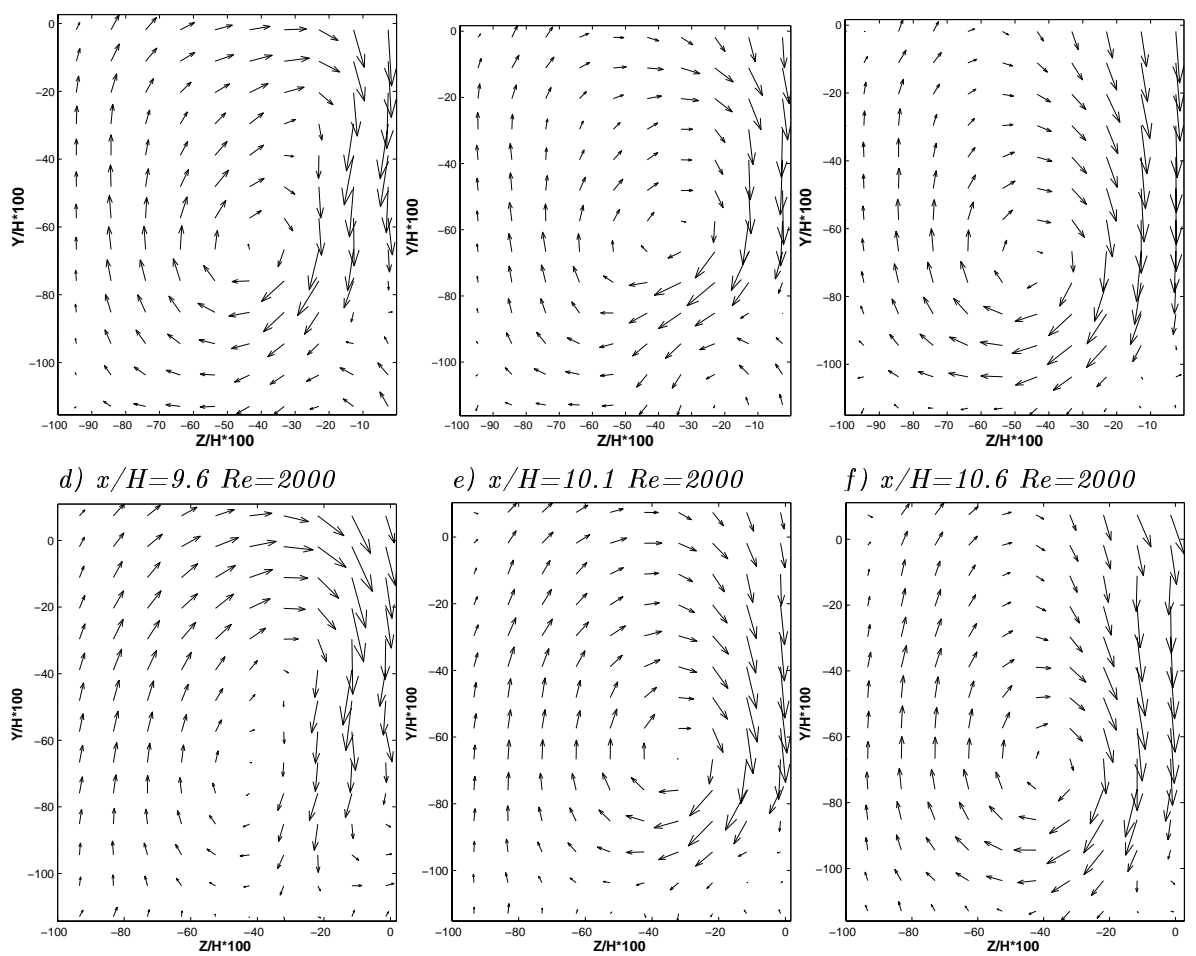

g) $x / H=9.6 R e=5000$
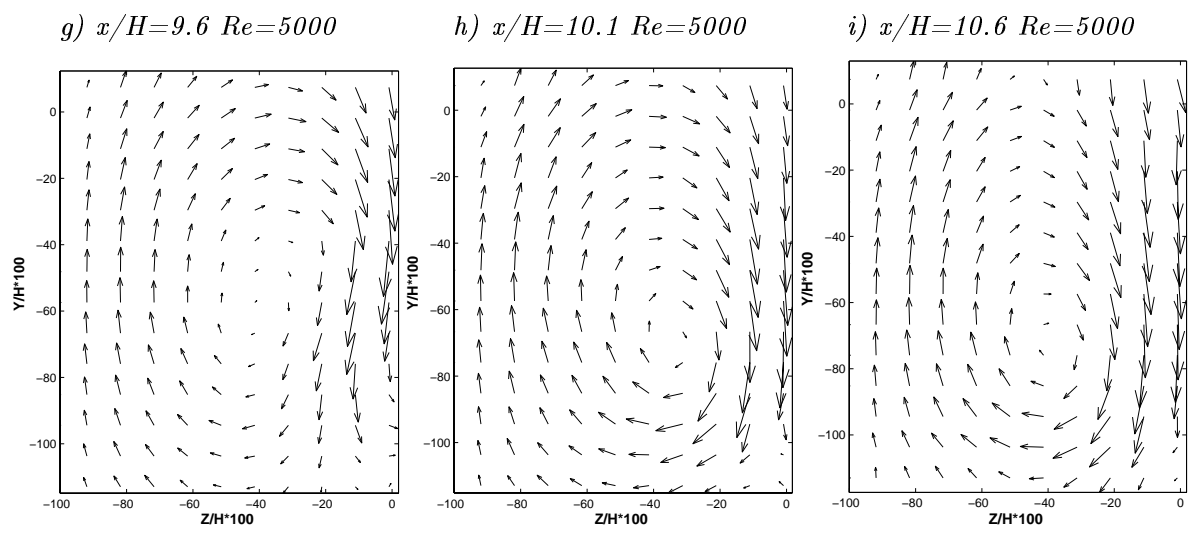

Figure 6: Mean velocity field in the cross section, for $R e=1000(a, b, c), R e=2000(d, e, f)$ and $R e=5000$ $(g, h, i)$ behind the $2^{\text {nd }}$ column at three streamwise cross sections $(O z, O y)$ 
a) $\frac{v^{\prime}}{U_{q}} \times 100$

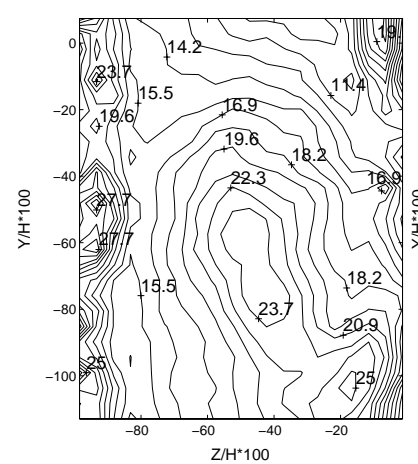

b) $\frac{u^{\prime}}{U_{q}} \times 100$

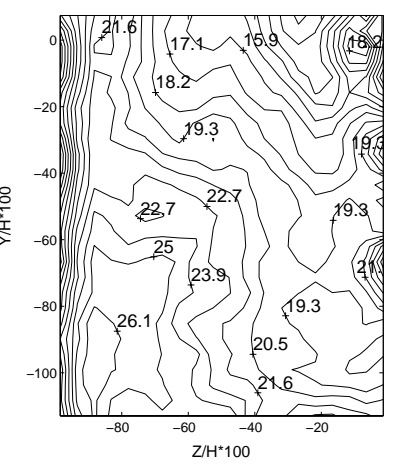

Figure 7: Iso-values (\%) of rms vertical (a) and rms longitudinal (b) velocity components, for Re=2000, behind the $2^{\text {nd }}$ column, at $\frac{x}{H}=11.1$ 
PSD (arbitrary units)

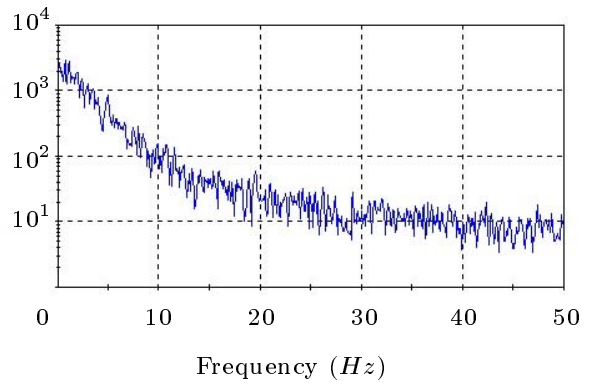

Figure 8: Example of Power Spectral Density of the transverse velocity fluctuations $v^{\prime}$, recorded at $\frac{x}{H}=19.1, \frac{y}{H}=-48.1, \frac{z}{H}=-82.4$, for $R e=2000$, with a sampling frequency of $100 \mathrm{~Hz}$ 


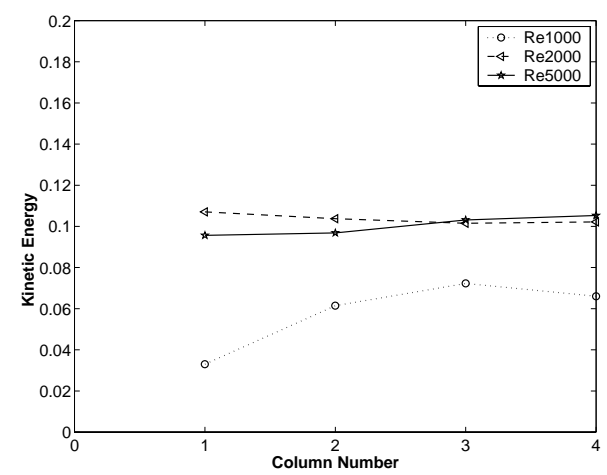

Figure 9: Average kinetic energy of the fluctuating motion $\frac{k}{\frac{1}{2} U_{q}^{2}}$ behind successive generators, at $\frac{d}{H}=2.4$ downstream of each generator 


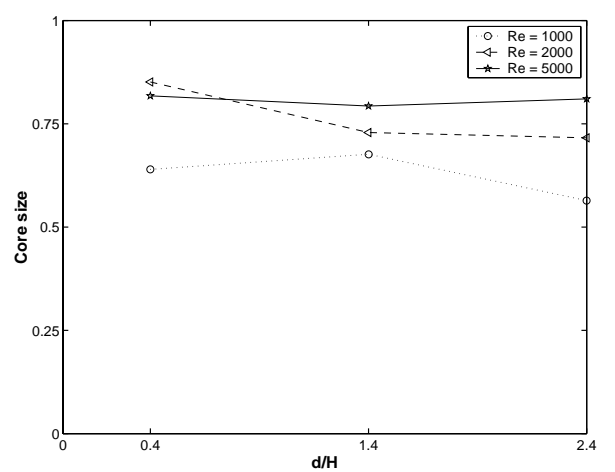

Figure 11: Vortex core size $\frac{2 a}{H}$ downstream of the $2^{\text {nd }}$ column 


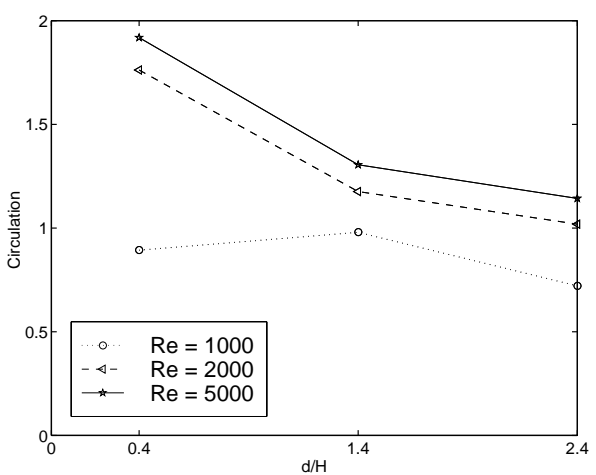

Figure 12: Vortex intensity $\frac{\Gamma}{U_{q} H}$ downstream of the $2^{\text {nd }}$ column 
